\title{
O COTIDIANO DA FAMÍLIA COM FILHOS PORTADORES DE FIBROSE CÍSTICA: SUBSÍdIOS PARA A ENFERMAGEM PEDIÁTRICA
}

Maria Cândida de Carvalho Furtado ${ }^{1}$ Regina Aparecida Garcia de Lima $^{2}$

Furtado MCC, Lima RAG. O cotidiano da família com filhos portadores de fibrose cística: subsídios para a enfermagem pediátrica. Rev Latino-am Enfermagem 2003 janeiro-fevereiro; 11(1):66-73.

A fibrose cística é uma doença genética caracterizada pelo aumento na produção de muco que, depositado em alguns órgãos, causa: doença pulmonar obstrutiva crônica, insuficiência pancreática e nível elevado de eletrólitos no suor. Pretendese, neste estudo, descrever o cotidiano de famílias com filhos portadores de fibrose cística nos aspectos relacionados à repercussão da doença crônica na dinâmica familiar. Para tanto, realizou-se um estudo qualitativo, com coleta de dados empíricos a partir de entrevistas com 14 famílias de pacientes menores de 18 anos em tratamento no Hospital das Clínicas da Faculdade de Medicina de Ribeirão Preto, da Universidade de São Paulo. Dos dados emergiram os seguintes temas: envolvimento da família, aceitação/entendimento da doença e enfrentando desafios. Identificamos uma vida de dependência relacionada à doença, com desgaste físico e emocional, tanto da criança quanto da família. Implicações para a enfermagem: constituição de modelos assistenciais que tenham a família como foco de atenção, considerando meio ambiente, estilo de vida e promoção à saúde como seus fundamentos básicos.

DESCRITORES: fibrose cística, família, enfermagem pediátrica

\section{THE ROUTINE OF FAMILIES WITH CHILDREN BEARING CYSTIC FIBROSIS:} SUBSIDIES FOR PEDIATRIC NURSING

Cystic Fibrosis is a genetic disorder characterized by the increase in the production of mucus whose accumulation on some organs causes: chronic obstructive pulmonary disease, pancreatic insufficiency and a high level of electrolytes in sweat. This study aims at describing the routine of families with children bearing cystic fibrosis as to the aspects concerning the effects of that chronic disease on the family dynamics. To that end, a qualitative study was conducted. Empirical data were collected by means of interviews with the families of patients aged less than 18 years old undergoing treatment at Hospital das Clínicas da Faculdade de Medicina de Ribeirão Preto da Universidade de São Paulo. The following themes emerged from the collected data: family involvement, acceptance/understanding of the disease and coping with challenge. A dependent life was identified which was related to the fact that the disease caused physical waste and emotional disturbance for both the child and the family. Implications for nursing: constitution of care models focusing on the family by considering the environment, life style and health as its basic fundamentals.

DESCRIPTORS: cystic fibrosis, family, pediatric nursing

\section{EL COTIDIANO DE LA FAMILIA CON HIJOS PORTADORES DE FIBROSIS QUíSTICA: APORTES PARA LA ENFERMERÍA PEDIÁTRICA}

La fibrosis quística es una enfermedad genética caracterizada por el aumento en la producción de moco que es depositado en algunos órganos causando: enfermedad pulmonar obstructiva crónica, insuficiencia pancreática y nivel elevado de electrolitos en el sudor. El objetivo de este estudio es describir el cotidiano de las familias con hijos portadores de fibrosis quística en los aspectos relacionados a la repercusión de la enfermedad crónica en la dinámica familiar. Por lo tanto, se realizó un estudio cualitativo, a través de la recolección de datos empíricos a partir de entrevistas con 14 familias de pacientes menores de 18 años en tratamiento en el Hospital Clínicas de la Facultad de Medicina de Ribeirão Preto de la Universidad de São Paulo. De los datos emergieron los siguientes temas: compromiso de la familia, aceptación/entendimiento de la enfermedad y enfrentamiento de desafíos. Identificamos una vida de dependencia relacionada con la enfermedad y desgaste físico y emocional del niño y de la familia. Implicaciones para la enfermería: constitución de modelos asistenciales que tengan la familia como foco de atención, considerando el medio ambiente, estilo de vida y promoción a la salud como sus fundamentos básicos.

DESCRIPTORES: fibrosis quística, familia, enfermería pediátrica

\footnotetext{
${ }^{1}$ Mestranda do Programa de Pós Graduação em Enfermagem em Saúde Pública, Especialista em Laboratório, Seção de Apoio Laboratorial, email: mariacandida@netsite.com.br; ${ }^{2}$ Orientadora, Professor Doutor, e-mail: limare@eerp.usp.br. Escola de Enfermagem de Ribeirão Preto da Universidade de São Paulo, Centro Colaborador da OMS para o desenvolvimento da pesquisa em enfermagem
} 
INTRODUÇÃO

$\boldsymbol{A}$ fibrose cística ou mucoviscidose é uma doença sistêmica, hereditária, autossômica recessiva, crônica e progressiva; é comum na raça branca e atinge igualmente ambos os sexos. O paciente portador dessa doença apresenta secreções mucosas espessas e viscosas, obstruindo os ductos das glândulas exócrinas, que contribuem para o aparecimento de três características básicas: doença pulmonar obstrutiva crônica, insuficiência pancreática com má digestão/má absorção e conseqüente desnutrição secundária, além de níveis elevados de eletrólitos no suor ${ }^{(1-3)}$.

A Fibrose Cística atinge aproximadamente 1:2.500 crianças nascidas-vivas da raça branca, incidência que diminui para indivíduos da raça negra na proporção 1:17.000 ${ }^{(4)}$. A expectativa de vida tem crescido nos últimos anos com os avanços tecnológicos que auxiliam no diagnóstico e terapêutica. Muitos autores estimam uma sobrevida em torno de 25 a 30 anos $^{(5-7)}$. Para alcançar essa sobrevida, o diagnóstico deve ser o mais precoce possível, devendo o tratamento, desde cedo, ser conduzido por profissionais adequadamente treinados ${ }^{(8)}$. Outros fatores também podem influenciar as taxas de sobrevida desses pacientes, quais sejam: melhor suporte nutricional; terapia antibiótica precoce e mais agressiva; desenvolvimento de centros de referência para diagnóstico e tratamento da doença cujo objetivo principal seja a melhoria na qualidade de vida desses pacientes, além da existência de suporte médico e promoção da educação dos pacientes e suas famílias ${ }^{(9)}$.

\section{REPERCUSSÕES DA DOENÇA CRÔNICA INFANTIL SOBRE A FAMÍLIA}

O estudo sobre família, neste contexto, surge da necessidade de explicitar sua atuação frente à doença crônica que afeta um de seus membros.

Para que possamos compreender o significado da palavra família é necessário defini-la, e dentre as várias definições, citamos a seguinte: que são relacionamentos em que pessoas vivem juntas, estão comprometidas, formando uma unidade econômica, cuidam dos mais jovens e se identificam entre si e no grupo a que pertencem ${ }^{(10)}$. Diante das diferentes definições encontradas, escolhemos esta, pois se refere às situações que vivenciam e que nos fazem compreender o significado que as fazem perceber que são famílias.
Até pouco tempo, as famílias eram afastadas das crianças que se encontravam internadas em verdadeiras situações de isolamento, pois acreditava-se que, assim, evitava-se a disseminação das infecções. As visitas eram programadas, e o pais não podiam, na maioria da vezes, entrar no local onde o filho encontrava-se internado. A partir da promulgação da lei no 8069, em 1990, que regulamenta o Estatuto da Criança e do Adolescente (ECA) $)^{(11)}$, as crianças passaram a ter o direito do acompanhamento do pai ou responsável durante as internações.

$\mathrm{Na}$ atualidade, observa-se um movimento de incentivo à participação e envolvimento da família no cuidado, identificando o importante papel que assume no processo saúde/doença/cuidar ${ }^{(12)}$. Neste estudo, conhecer a família, a sua constituição, a relação entre os membros que a compõem, quem são, como é seu cotidiano, como reestruturam a vida familiar frente à condição crônica de seu(s) filho(s) e como participam do cuidado prestado, é de fundamental importância.

A existência de uma doença crônica afeta toda a família e gera momentos difíceis com avanços e retrocessos nas relações entre seus membros ${ }^{(13)}$. O cuidado à criança com doença crônica consome da família energia e tempo e retira sua privacidade; pode também provocar isolamento social e emocional. Em contrapartida, se a família tem conhecimento sobre a doença, o tratamento e os recursos disponíveis, seus níveis de estresse e ansiedade podem diminuir significativamente ${ }^{(14-}$ 15).

Pelo fato de essa doença atingir variados sistemas, é necessário um cuidado complexo e contínuo para a promoção de uma condição estável do paciente. Essa atenção em tempo integral faz com que os responsáveis pelo cuidado necessitem conhecer a doença e a terapêutica para possibilitar a assistência desejada, No entanto, apenas o saber clínico é insuficiente. É importante conhecer como e quanto a família foi afetada em todos os seus aspectos (sociais, emocionais e financeiros).

\section{OBJETIVOS}

Traçamos como objetivo deste estudo: descrever o cotidiano das famílias com filhos portadores de fibrose cística, buscando apreender aspectos necessários para implementação de uma assistência visando à melhoria na qualidade de vida do binômio pacientes/famílias. 
O CAMINHO METODOLÓGICO

Este é um estudo-descritivo exploratório, de natureza qualitativa, que busca examinar o cotidiano das famílias de crianças/adolescentes com fibrose cística, com o fim de identificar a repercussão da condição crônica na dinâmica familiar. A coleta de dados empíricos foi realizada a partir de entrevistas com 14 famílias de pacientes portadores de fibrose cística, menores de 18 anos.

\section{Local do estudo}

O estudo foi realizado no Hospital das Clínicas da Faculdade de Medicina de Ribeirão Preto, da Universidade de São Paulo (HCFMRP-USP), instituição hospitalar de nível terciário e de referência para várias patologias, onde os pacientes portadores de fibrose cística são acompanhados, tanto no Ambulatório como na Unidade de Internação Pediátrica.

\section{Participantes}

Após aprovação do Comitê de Ética em Pesquisa do HCFMRP-USP, ocorrida em novembro de 1999, os participantes foram recrutados mediante informação obtida no Serviço de Arquivo Médico e Estatístico (SAME). Para tanto, solicitamos que o SAME nos fornecesse uma listagem dos paciente portadores de fibrose cística, menores de 18 anos, diagnosticados e em acompanhamento no referido hospital, desde 1982. A escolha por esse período deve-se ao início da informatização do sistema de registros dos pacientes, pelo hospital. Encontramos um total de 82 pacientes e, dentre esses, 13 óbitos constatados. Dos 69 pacientes restantes, apenas 28 continuam em acompanhamento neste hospital, dentre os quais 25 são menores de 18 anos. Optamos, portanto, pela totalidade de pacientes.

A partir dessas crianças/adolescentes, chegamos às suas famílias e constatamos que quatro delas tinham dois filhos com fibrose cística, dessa forma, ao invés de 25 famílias, participaram do estudo apenas 21. Dessas, apenas uma família recusou-se a participar da pesquisa, uma outra foi consultada a respeito da sua participação, aceitou, mas, antes da entrevista previamente marcada, a criança foi a óbito, e a mãe recusou-se a falar sobre a doença da filha. Não conseguimos localizar seis famílias, por estas não comparecerem aos retornos marcados ou por não terem sido contactadas durante a busca. As demais não se opuseram a participar do estudo, permitindo que a entrevista fosse gravada. Assim, entrevistamos 14 famílias, totalizando 18 pacientes portadores de fibrose cística. Contamos com a participação de todas as mães (14) e, além delas, participaram das entrevistas cinco pais, uma prima e uma tia.

O respeito ao anonimato e o sigilo são particularmente importantes, pois, em pequenos espaços sociais ou grupos reduzidos, em geral, por meio de alguns descritores, é possível identificar os sujeitos. Para evitar que a identificação fosse feita por meio da informação, numeramos as entrevistas em ordem crescente, tendo a primeira recebido a codificação $E 1$ e, assim, sucessivamente. Para obtermos uma visão mais global dessa fase, registramos as principais impressões de cada entrevista, em um diário de campo, dedicando especial atenção para a comunicação não-verbal, como entonação, risos, pausas, hesitações. Registramos, também, a hora do início e do término de cada entrevista.

As identificações das crianças participantes deste estudo seguiram as numerações das entrevistas das famílias. Portanto, quando a família tinha mais de uma criança com fibrose cística, elas foram identificadas na mesma entrevista (ex.: E5/6).

Coleta de dados

Foram utilizadas duas fontes de coleta de dados: entrevista semi-estruturada e levantamento de prontuários. A estrutura básica da entrevista foi constituída por duas partes: identificação da família e questões diretivas. A primeira contém questões relacionadas aos componentes do grupo familiar e condições sociodemográficas de seus membros, como sexo, idade, escolaridade, renda familiar, ocupação e responsável pelo cuidado. Na segunda parte, as famílias descreveram o seu cotidiano nos aspectos relacionados à dinâmica familiar no cuidado ao(s) filho(s) com fibrose cística. Para direcionar esse trabalho, elaboramos as seguintes questões: (1) Como é cuidar de um criança com Fibrose Cística? Descreva como é o diaa-dia da família em relação ao cuidado; (2) Como vocês convivem com a doença, como reagem e se sentem?; (3) Como cada membro da família se organiza (como cada um contribui) para ajudar no cuidado domiciliar?; (4) Quem tem mais facilidade ou mais dificuldade no cuidado da criança/adolescente?

Os dados foram coletados no período de maio de 2000 a janeiro de 2001. 
Análise dos dados

Iniciamos a análise dos dados com a sua ordenação ${ }^{(16)}$ a partir da transcrição integral das fitas e da leitura do material empírico, preservando as falas dos entrevistados. No total, foram 12 horas de gravação e, após a transcrição das fitas, obtivemos 88 páginas de informações colhidas das famílias de pacientes portadores de fibrose cística, por ocasião das entrevistas.

Inicialmente, selecionamos partes do material, agrupando-as de acordo com a semelhança dos relatos dos entrevistados, a partir de recortes das entrevistas, buscando, posteriormente, as suas idéias relevantes. Esse material foi distribuído em um quadro, que demonstra, à esquerda, a fala dos entrevistados e, à direita, a codificação. Esses códigos foram ditados pelas idéias ou pelas próprias falas dos entrevistados, tendo o pesquisador o cuidado de registrar em negrito o que de mais relevante foi expresso por eles, como demonstrado a seguir.

\begin{tabular}{|l|c|}
\hline TRECHOS DAS ENTREVISTAS & CÓDIGOS \\
\hline "O meu dia-a-dia não é fácil, né, \\
porque tem sempre que estar por \\
conta dela...Fazer papinha, dar \\
remédio na hora certa, tudo certo, \\
essas coisas..." E2 \\
"Mas é cansativo...a gente tem \\
que doar...a gente doa a \\
vida...porque a gente tá sempre \\
abrindo mão(...) Quando você tem \\
uma criança com mucoviscidose \\
você vive abrindo mão". E16 \\
\hline "Eu que tenho que fazer \\
fisioterapia, eu que tenho que \\
cuidar, se interna, eu que fico \\
junto". E3 outros \\
"Eu cato tudo pra mim...em se \\
tratando de mucoviscidose \\
então..." E16 \\
\hline "Eu sei distingüir quando é uma \\
tosse fina, uma tosse que tá \\
infectada mesmo..." E1 \\
Aí foi indo até eu pegar prática(...) \\
Agora eu já acostumei...eu sei \\
quando ele tá bom, quando ele \\
não tá, o que eu faço, o que eu \\
posso fazer...já adquiri toda essa \\
força...esse conhecimento". E18
\end{tabular}

Figura 1 - Codificação dos dados coletados através das entrevistas com as famílias de crianças/adolescentes portadores de fibrose cística. Ribeirão Preto-SP, 2000

Mediante a releitura dos códigos encontrados, agrupamos os mesmos de acordo com sua familiaridade, surgindo, então, os subtemas. Após sua ordenação, passamos à construção dos temas, como nos mostra a figura a seguir.

\begin{tabular}{|c|c|c|}
\hline CÓDIGOS & SUBTEMAS & TEMAS \\
\hline $\begin{array}{l}\text { Abrindo mão de outros } \\
\text { papéis }\end{array}$ & \multirow{4}{*}{$\begin{array}{l}\text { Exclusividade da } \\
\text { atenção }\end{array}$} & \multirow{6}{*}{$\begin{array}{l}\text { Envolvimento } \\
\text { da família }\end{array}$} \\
\hline Sobrecarga materna & & \\
\hline $\begin{array}{l}\text { Aprendendo com a } \\
\text { doença }\end{array}$ & & \\
\hline Dependendo da mãe & & \\
\hline Não trabalhando & $\begin{array}{c}\text { Divisão do } \\
\text { trabalho/cuidado }\end{array}$ & \\
\hline $\begin{array}{l}\text { Mantendo a relação } \\
\text { igual }\end{array}$ & Relação entre irmãos & \\
\hline Aceitação & \multirow{2}{*}{$\begin{array}{l}\text { A doença crônica } \\
\text { afetando a família }\end{array}$} & \multirow{2}{*}{$\begin{array}{l}\text { Impacto da } \\
\text { doença }\end{array}$} \\
\hline $\begin{array}{l}\text { Tendo dois filhos } \\
\text { afetados }\end{array}$ & & \\
\hline Tendo ajuda de outros & Redes de apoio & \multirow[b]{2}{*}{$\begin{array}{l}\text { Enfrentando } \\
\text { desafios }\end{array}$} \\
\hline $\begin{array}{l}\text { Ajudando-se } \\
\text { Mantendo a } \\
\text { esperança }\end{array}$ & $\begin{array}{l}\text { Troca de experiências } \\
\text { promovendo o apoio } \\
\text { às famílias }\end{array}$ & \\
\hline
\end{tabular}

Figura 2 - Construção dos temas, a partir do agrupamento dos códigos encontrados nas entrevistas com as famílias de crianças/adolescentes portadores de fibrose cística. Ribeirão Preto-SP, 2000

Chegamos, assim, aos temas, partindo dos respectivos subtemas. Envolvimento da família teve como subtemas: exclusividade da atenção, divisão do trabalho/cuidado e relação entre irmãos; impacto da doença que partiu do subtema a doença crônica afetando a família e o tema enfrentando desafios que teve como subtemas: redes de apoio e troca de experiências promovendo o apoio às famílias.

\section{RESULTADOS EM DISCUSSÃO}

Dividimos a discussão dos resultados em duas partes; na primeira, apresentamos a caracterização das crianças/adolescentes portadores de fibrose cística e de suas famílias e, na segunda parte, discutiremos os temas levantados durante a análise do estudo.

Caracterização das crianças/adolescentes portadores de fibrose cística e de suas famílias

A partir da caracterização das crianças/ adolescentes portadores de fibrose cística atendidos no HCFMRP-USP, observamos que metade dos pacientes é do sexo feminino e outra metade do sexo masculino. A idade, na data do diagnóstico, variou, sendo que um maior 
número de casos foi diagnosticado antes de um ano de idade, constatação também descrita por outros autores ${ }^{(9)}$. A maioria das crianças é procedente de outras cidades do estado de São Paulo e de outros estados, pelo fato de esse hospital ser referência no atendimento de pacientes portadores de fibrose cística. Percebemos, também que mesmo pacientes que tiveram poucas internações ou não as tiveram, apresentam um número significativo de retornos ambulatoriais, fato que nos mostra que, em sendo esta, uma doença crônica, requer um seguimento contínuo, mesmo que a criança não necessite de internação.

Do total de famílias de crianças/adolescentes portadores de fibrose cística entrevistadas (14), oito crianças são irmãs, sendo pertencentes à quatro famílias. Algumas considerações sobre o caráter genético da doença já haviam sido feitas ${ }^{(7,17)}$, e nossos dados reforçam a necessidade de acompanhamento e orientação dessas famílias quanto à possibilidade do nascimento de um filho doente. Da escolaridade dos pacientes estudados, encontramos que os que estão em idade escolar possuem um acompanhamento adequado, sem repetência ou desistência, o que mostra a tentativa de a família tornar a vida no núcleo familiar a mais próxima possível do normal.

A maioria dos pais dessas crianças/adolescentes possui entre 31 e 40 anos e com o ensino fundamental incompleto, o que compromete a renda familiar, que, na maioria, varia entre 1 e 2 salários mínimos. A condição socioeconômica mais baixa tem como reflexo a dificuldade na aquisição de medicamentos de alto custo, necessários durante o tratamento, como também de suplementação alimentar, e essas dificuldades geram mais ansiedade ainda, quando no núcleo familiar há mais de uma criança acometida pela doença ${ }^{(14,17)}$. Das famílias entrevistadas, doze mães relataram não exercerem atividades extralar, permanecendo com seus filhos em casa, com impossibilidade de sair para trabalhar devido tempo exigido no cuidado ao filho doente. Como responsável pelo cuidado ao filho doente, em todas as entrevistas, as mães responderam serem elas as que o administram.

Convivendo com a fibrose cística

A partir das entrevistas com famílias de crianças/ adolescentes portadores de fibrose cística foi possível a apreensão dos temas que discutiremos a seguir.
Envolvimento da família

Pelo fato de a fibrose cística atingir variados sistemas do corpo humano, ela exige um cuidado complexo e uma atenção contínua para promoção de uma condição clínica estável do paciente; para tanto, é imprescindível o envolvimento da família, sob as mais diferentes maneiras. A família convive, desde a fase diagnóstica, com alterações em suas rotinas, voltando sua atenção para o filho doente. A família passa a viver sob forte ansiedade, que pode ser minimizada quando preparada para os cuidados domiciliares. Esse plano de cuidados deve ser flexível, de modo que as atividades familiares sejam interrompidas com a menor freqüência possível.

Os cuidados do paciente com fibrose cística exigem dos pais e, principalmente do responsável pelo cuidado (caso representado geralmente pela mãe), um tempo maior do que aquele dispensado às outras atividades no âmbito familiar. Essa dedicação mostra-se como uma dificuldade pelo fato de a mãe ter, além desses cuidados, outras obrigações. É o que podemos observar nos relatos a seguir:

O meu dia-a-dia não é fácil, né, porque tem sempre que estar por conta dela...Fazer papinha, dar remédio na hora certa, tudo certo... E2

É muito serviço...é um dilema de serviço...não pára. Tem o serviço da gente(...) se fosse só por conta dela... E13/14

Os pais vêem suas vidas envolvidas pela doença e o mesmo ocorre com toda a família ${ }^{(13,18-19)}$ e esse envolvimento reflete uma necessidade de atenção especial, alterando o ritmo familiar. Percebemos que o cuidado centraliza-se mais na figura materna, pois ela aprende como cuidar e preocupa-se em executar corretamente esse cuidado. Pela convivência diária com a doença, aumenta seu conhecimento sobre a situação do filho, tendo uma visão mais apurada com relação aos aspectos clínicos e emocionais apresentados pela criança, assim como passam a conhecer suas reações e necessidades.

Aí foi indo até eu pegar prática(...)Agora eu já acostumei...eu sei quando ele tá bom, quando não tá(...)já adquiri toda essa força...esse conhecimento. E18

As crianças doentes muitas vezes determinam a exclusividade do cuidado materno por não permitirem que outras pessoas o façam. Do mesmo modo, a dependência da criança é reforçada pelo vínculo mãe-filho. Para ele, ter 
a mãe como cuidadora primária, aumenta sua segurança e confiança, mesmo quando não aceita alguma intervenção.

Sou mais eu pra mexer com ele. Ele não aceita que seja outra pessoa(...) ele só aceita que seja eu. E4

Uma das mudanças decorrentes da doença crônica no núcleo familiar refere-se ao trabalho das mães fora de casa, no caso das mães. A mulher, que divide os papéis de mãe e trabalhadora, tem seu lado maternal e cuidador revelado de maneira intensa, quando se trata de doença no âmbito familiar. Nesses casos, ela deixa o trabalho fora de casa para segundo ou até mesmo para último plano, ao se defrontar com a necessidade de cuidar de um filho doente. Sua atuação no lar impede o exercício de atividades externas a ele e, como mulher e mãe, à frente do cuidado do filho doente, ela acaba não tendo como dividir seu tempo entre as duas atividades, pois a exigência de atenção integral ao cuidado ${ }^{(13)}$ impossibilita que tenha outra atividade, que não a do lar.

Não posso trabalhar fora...tem que cuidar só dele(...)não tem tempo pra mais nada. E3

Quando discutimos uma doença crônica, é inviável pensarmos somente nos aspectos que influenciam diretamente a pessoa doente. A doença faz parte do contexto familiar e, assim, modifica as relações de todos os seus membros, mesmo entre os irmãos, a qual não pode ser negligenciada, já que a criança estabelece com os irmãos um convívio de parceria e aprendizagem. Observamos, neste estudo, que a relação entre os irmãos é expressa sempre como normal, comum, pelos entrevistados, e que os pais são os responsáveis pela manutenção desse "estado de normalidade", o qual pode se desequilibrar pela maior atenção da mãe ao filho doente e por não sabermos como os irmãos são afetados nessa relação, é preciso torná-la a mais próxima possível do normal $^{(20)}$.

Eu faço tudo o que dá pra fazer pros dois, eu não tenho limites. Quando brigam...eles já sabem lá em casa, se tiver que ficar de castigo, fica todo mundo. E1

Impacto da doença

O contato inicial com a doença gera nos pais a ansiedade e o medo. Muitas vezes, eles expressam a incredulidade com relação ao diagnóstico, o receio da perda do filho de imediato. Essas fases enfrentadas pela família, descritas como as reações iniciais dos pais (choque, incredulidade, confusão, medo, raiva, conflito, tensão), apresentam duração variável e uma negação prolongada, têm reflexo em toda a família, impedindo uma adaptação bem sucedida ${ }^{(15,21)}$.

As famílias cujos filhos são portadores de fibrose cística apresentam necessidades emocionais semelhantes àquelas manifestadas por famílias que têm em seu seio crianças com outras doenças crônicas, e um motivo que se constitui fonte constante de ansiedade para os pais e para a criança, é a presença do temor da morte ${ }^{(22)}$.

Eu tava com medo, porque na minha cabeça eu morria de medo era de perder ele(...)eu achava que essa doença ia levar meu filho de uma hora pra outra. E18

O desconhecimento da doença faz com que as famílias simulem situações futuras que as impedem de se adaptar à nova condição e de compreender o significado de cuidado e atenção contínua. A aceitação da doença ocorre com o aumento do conhecimento que dela se adquire, de como cuidar da criança e como essa pode reagir. A família passa a se sentir segura a partir do momento em que começa a aprender a lidar com a doença do filho e sente que está realizando um bom cuidado, com resultados visíveis, ou seja, com melhora no estado clínico da criança.

Eu não aceitava de jeito nenhum(...) eu não admitia que meu filho era doente(...)Aceitar?...o momento pra mim foi quando eu consegui, senti a capacidade de entender o que era a mucoviscidose, conhecer a doença. Conhecer assim...os cuidados...como lidar com a criança... E 18

\section{Enfrentando desafios}

Os pais relatavam, no decorrer das entrevistas, 0 quanto era importante para eles e toda a sua família o apoio recebido de diferentes grupos (família, amigos, equipe de saúde) para o enfrentamento das situações vivenciadas no cuidado cotidiano da criança/adolescente portador de fibrose cística. É a partir das redes de apoio e da troca de experiência promovendo esse apoio que as família desenvolvem meios para superar as dificuldades que encontram nessa assistência.

As famílias podem, inicialmente, enfrentar sozinhas a doença e o que ela traz consigo: a dependência de uma atenção contínua, cuidados com a alimentação, tratamento medicamentoso e também a constante preocupação com o estado clínico do doente. Mas o 
contexto que as envolve vai além dos limites do lar. A rede social mostra-se como fonte necessária para os enfrentamentos cotidianos.

Grupos de pais com filhos em situações semelhantes têm surgido para facilitar o enfrentamento das famílias frente à condição crônica de seus filhos. Esses grupos, geralmente, são formados por pais veteranos, experientes no cuidado de seus filhos, que compartilham com pais mais novos alguns meios de enfrentamento de situações que emergem no cuidado diário de pacientes com condição crônica ${ }^{(18)}$. Esses grupos acabam funcionando como complemento ao apoio profissional.

Percebemos, no decorrer deste estudo, que a maior necessidade das famílias com filhos portadores de fibrose cística pode ser sanada com a ajuda advinda de outros membros da família, de outras famílias em situações semelhantes e também da equipe de saúde.

Se não fosse o grupo de mães com que a gente reúne, conhecesse outras mães, nossa, era muito mais doloroso(...) se tinha que conversar com outras mães e falar não, é assim, isso acontece, você fica muito mais...sabe...tranqüila, mais confiante. Passa uma confiança muito grande. E3

A ajuda prestada pelas famílias que estão com seus filhos em situações semelhantes é mencionada nas entrevistas como expressão de alívio, por encontrar pessoas que possuem as mesmas vivências ${ }^{(13,18)}$. As mães nunca desistem; perseveram para aliviar a ansiedade causada pela doença e seus cuidados. Aproveitam as oportunidades em que estão próximas para poder passar umas às outras suas experiências, como vivenciaram e enfrentaram situações que hoje parecem não as assustar mais.

Precisa lutar, fazer, como se fala...uma ajudar a outra...se tiver problemas, dúvidas...uma que dá apoio à outra às vezes muda muito a cabeça...aceita melhor. E1

No começo é difícil. Hoje a gente pode passar pras outras mães que não é um bicho de sete cabeças. E18

Atualmente, existem associações que promovem o apoio às famílias com doentes crônicos, a exemplo da ABRAM (Associação Brasileira de Apoio ao Mucoviscidótico). Ampliar o apoio, buscando ajudar as famílias de maneira a ultrapassar a questão biológica é um papel que o enfermeiro pode e deve estar desempenhando. Isso o tornará mais apto a adquirir conhecimento e buscar, juntamente com as famílias, melhorar o cuidado a ser prestado.

\section{CONSIDERAÇÕES GERAIS}

Dos resultados alcançados, percebemos que a doença crônica afeta toda a família, sendo que a sobrecarga recai sobre a figura materna, tanto no que diz respeito aos cuidados prestados como na atenção constante na evolução desse paciente.

A mãe abre mão de outras atividades e papéis para atender seu filho, acarretando, com isso, certas dificuldades no relacionamento intra e extrafamiliar. Sua vida particular pode acabar sendo prejudicada em detrimento do filho doente. Ressaltamos que a intensidade e continuidade do cuidado geram um maior conhecimento da doença que tem, como aspecto positivo, a diminuição da ansiedade gerada pela doença crônica. Contrapondo a isso, aparece a falta de conhecimento no início da convivência com a doença que afeta a família por não conseguir assistir a criança da maneira desejada.

As redes de apoio aparecem como uma necessidade mais do que eminente, visto que as famílias dividem experiências semelhantes, adquirem segurança na condução do tratamento do filho, reflexo que acaba beneficiando todos os membros da família.

Proporcionar e possibilitar encontros de pais que experimentam situações semelhantes fortalece cada família, com reflexos positivos na condução do tratamento da criança. Diminuindo a ansiedade dos pais mediante 0 conhecimento da doença, tornando-os capazes de realizar uma assistência adequada, transforma-os em multiplicadores de experiências positivas e de melhoria na qualidade de vida desejada para o filho.

Quando, como enfermeiros, assistimos a um paciente com doença crônica, temos que pensar, também, que ele carrega consigo toda uma bagagem de vivências, relações, sensações, sentimentos. Pensando assisti-lo de uma maneira mais ampla, buscamos também perceber a sua família como parte de todo o contexto que envolve esse paciente.

Considerando que a Fibrose Cística é uma doença que acomete os pacientes, em geral, na infância, a enfermagem deve estar ainda mais aberta a fazer a família participar junto à doença da criança. Os pais que vivenciam a doença do filho são, potencialmente, os melhores indicadores de caminhos de intervenções que a enfermagem pode seguir.

Identificando como a criança e sua família convivem 
com a doença, qual a repercussão da doença na dinâmica intrafamiliar e/ou a contribuição do ambiente na evolução da enfermidade, o enfermeiro poderá estar formando vínculos com familiares de crianças e adolescentes

\section{REFERÊNCIAS BIBLIOGRÁFICAS}

1. Barbieri D, Quarentei G. Mucoviscidose (Fibrose Cística). In: Marcondes E. Pediatria básica. São Paulo (SP): Sarvier; 1994. p.1206-12.

2. Colin AA, Whol MEB. Cystic fibrosis. Pediatr Rev 1994; 15(5):192-200.

3. Bentley $P$. Understanding cystic fibrosis, improving life expectancy. Nurs Times 1999; 95(43):50-1.

4. Nelson WE, Vaughan VC, Mckay RJ. Tratado de Pediatria. 14르 ed. Rio de Janeiro (RJ): Guanabara Koogan; 1994.

5. White KR, Munro CL, Pickler RH. Therapeutic implications on recents advances in Cystic Fibrosis. MCN 1995; 20:304-8. 6. Scatollin I, Beier S, Blacher J, Laurent MC, Santos MIS. Desenvolvimento da atenção integral à criança com fibrose cística. In: Ceccim RB, Carvalho PPA. Criança hospitalizada: atenção integral como escuta à vida. Porto Alegre (RS): Editora da Universidade/UFRGS; 1997. p.91-5.

7. Bush A, Wallis C. Time to think again: Cystic Fibrosis is not "all or none" disease. Pediatr Pulmonol 2000; 39:139-44.

8. Reis FC, Rozov T. Fibrose Cística. In: Sant'anna CC, Leite AJM, coordenadores. Manual de Doenças Respiratórias na Infância. Rio de Janeiro (RJ): Sociedade Brasileira de Pediatria/Comitê Pneumologia; 1995.

9. Reis FJC, Damasceno N. Fibrose Cística. J Pediatr 1998; 74 (Supl 2):76-94.

10. Kendall D, Linden R, Murray JC. Sociology our times: the essencial.Toronto: ITP Nelson; 1998.

11. Ministério da Saúde (BR). Estatuto da Criança e do Adolescente. Brasília (DF): Ministério da Saúde; 1991.

12. Angelo M. Com a família em tempos difíceis: uma perspectiva de enfermagem. [Tese]. São Paulo (SP): Escola de Enfermagem/USP; 1997.

13. Anders JC. A família na assistência à criança e ao adolescente submetidos ao transplante de medula óssea: fase pós-TMO. [dissertação]. Ribeirão Preto (SP): Escola de Enfermagem de Ribeirão Preto/USP; 1999.

14. Beresford B. Resources and strategies: how parents cope with the care of disable child. J Child Psychol Psychiatr 1994; 35:171-209.

15. Jerret M, Costello E. Gainning control: parents' experience of accomodating children's asthma. Clin Nurs Res 1996; 5(3):294-308.

16. Bogdan R, Biklen S. Investigação qualitativa em educação: uma introdução à teoria e aos métodos. Porto (Portugal): Porto Editora; 1994.

17. Sagesse ESR. Relação médico-paciente em pediatria uma experiência com pacientes de mucoviscidose. J Bras Psiq 1983; 32(2):77-86.

18. Darbyshire $\mathrm{P}$, Morrison $\mathrm{H}$. Empowering parents of children with special needs. Nurs Times 1995; 91(32):26-8.

19. Callery C. Maternal knowledge and professional knowledge: co-operation and conflit in the care of sick children. Int J Nurs Stud 1997; 34(1):27-34. portadores de fibrose cística e, por meio destes, auxiliálos na convivência diária, na compreensão e no enfrentamento da doença, estabelecendo meios para facilitar o processo de tratamento.

20. Hayes VE. The impact of a child's chonic condition on the family system. [Doctor of Philosophy in Nursing]. San Francisco: University of California; 1992.

21. Wiserman $\mathrm{H}$. The effects of chronic childhood illness on families. Nurs Times 1996; 92(27):44-6.

22. Wong DL. Enfermagem pediátrica: elementos essenciais à instrumentação efetiva. $5^{a}$ ed. Rio de Janeiro (RJ): Guanabara Koogan; 1999. 